\title{
Comparative transcriptional and functional profiling of clear cell and papillary renal cell carcinoma
}

\author{
JULIA DIEGMANN ${ }^{1}$, STEFAN TOMIUK $^{2}$, JIMSGENE SANJMYATAV $^{3}$, KERSTIN JUNKER $^{3}$, \\ WINFRIED HINDERMANN ${ }^{4}$ and FERDINAND VON EGGELING ${ }^{1}$
}

\begin{abstract}
${ }^{1}$ Core Unit Chip Application (CUCA), Institute of Human Genetics and Anthropology, Friedrich-Schiller-University, 07740 Jena; ${ }^{2}$ Miltenyi Biotec GmbH, MACSmolecular Business Unit, Stöckheimer Weg 1, 50829 Köln; ${ }^{3}$ Department of Urology, Medical Faculty, Friedrich-Schiller-University, Lessingstr. 1, 07743 Jena; ${ }^{4}$ Institute of Pathology, Medical Faculty, Friedrich-Schiller-University, Ziegelmühlenweg 1, 07743 Jena, Germany
\end{abstract}

Received March 16, 2006; Accepted May 8, 2006

\begin{abstract}
Renal cell carcinoma (RCC) is known to effectively prevent immune recognition. However, little is known about the mechanisms that underlie this phenomenon. Thus, the identification of immunogenic molecules associated with RCC and the elucidation of the corresponding signaling pathways are crucial to the development of effective treatments. We performed transcriptional and functional profiling with cDNA microarrays (1070 cDNA probes) on a total of 17 RCCs, 11 clear cell and 6 papillary, and on corresponding normal tissue. Samples were clustered based on their expression profiles. We found a total of 45 genes to be regulated equally by both tumor types compared to the normal tissue. A set of 13 differentially expressed genes was identified between the examined tumor subtypes. Functional analysis was performed for both gene sets and showed a significant enrichment of cell surface genes regulated in both tumor subtypes. Within these we found five surface marker genes to be upregulated (TNFRSF10B, CD70, TNFR1, PDGFRB, and BAFF) which are involved in immune responses via the regulation of lymphocytes and can also induce apoptosis. Their overexpression in both tumor subtypes suggests a possible involvement in the immune escape strategies of RCC. The combination of transcriptional and functional profiling revealed potential target molecules for novel therapy strategies that must be studied in more detail.
\end{abstract}

\section{Introduction}

Renal cell carcinoma (RCC) is the third most common tumor within urological oncology and comprises $2-3 \%$ of all

Correspondence to: Dr Ferdinand von Eggeling, Institut für Humangenetik und Anthropologie, CUCA, 07740 Jena, Germany

E-mail: fegg@mti.uni-jena.de

Key words: CD70, TNFSF7, cDNA-arrays, kidney cancer, pathway analysis, statistical analysis of microarrays malignancies. The most frequent histological subtypes of RCC are clear cell (80\%) and papillary (10\% to $15 \%)(1-3)$. Even though RCC is known to prevent immune recognition through induction of apoptosis in T-lymphocytes only a few genes, like FasL, are known to be involved in this phenomenon (4). In order to elucidate more mechanisms involved in RCC immune escape strategies, we compared two separate gene expression studies using cDNA arrays. The highly parallel analysis of gene expression has already been successfully applied for biomarker discovery $(5,6)$. However, it is possible to extract even more information from the expression profiles generated via functional profiling. This method was developed to translate the typical list created by microarrays of tens or hundreds of genes found to be differentially expressed into a better understanding of the biological phenomena involved (7).

Functional profiling has been successfully used to elucidate gene expression patterns in cancer on the basis of gene expression studies $(8,9)$. Nevertheless, to date no study has been carried out to identify patterns enabled in RCC. We therefore compared two independent gene expression datasets for papillary (pRCC) and clear cell (ccRCC). The ccRCC dataset was reported in an earlier study (10). For the pRCC, a separate gene expression study was carried out. We found several genes to be commonly and differentially expressed and subjected them to a functional annotation analysis. The functional annotations revealed an enrichment of immunogenic molecules that were regulated in both tumor types. These genes are likely to play a role in RCC immunogenicity and can serve as target molecules for new therapies. These results extend our existing knowledge about the organizational pattern of gene expression in human RCC and lead to promising target molecules for more effective therapies.

\section{Materials and methods}

Tissue samples. Samples from 11 patients with ccRCC and 6 patients with $\mathrm{pRCC}$, containing normal non-tumor and tumor tissue were obtained from fresh nephrectomy specimens (Table I). The samples were snap frozen in liquid nitrogen and stored at $-80^{\circ} \mathrm{C}$ at the Department of Urology of the 
Table I. Overview of all examined patients with corresponding tumor relevant information. ${ }^{\mathrm{a}}$

\begin{tabular}{|c|c|c|c|c|c|c|}
\hline Patient & Cell type & Appearance & Grading & $\begin{array}{l}\text { pT (extension } \\
\text { of primary tumor) }\end{array}$ & $\begin{array}{l}\text { pN (infiltration } \\
\text { of lymph nodes) }\end{array}$ & $\begin{array}{c}\mathrm{pM} \\
\text { (metastasis) }\end{array}$ \\
\hline 1 & Papillary & Papillary & 2 & $1 \mathrm{a}$ & - & - \\
\hline 2 & Papillary & Papillary & 3 & 1 & 0 & - \\
\hline 3 & Papillary & Papillary & 2 & $1 b$ & 0 & $\mathrm{x}$ \\
\hline 4 & Papillary & Tubulo-papillary & - & 1 & - & - \\
\hline 5 & Papillary & Papillary & - & $3 a$ & - & - \\
\hline 6 & Papillary & Tubulo-papillary & 1 & $3 a$ & 0 & $\mathrm{x}$ \\
\hline 7 & Clear-cell & - & - & 1 & - & - \\
\hline 8 & Clear-cell & Azinary, tubulary & 2 & - & - & - \\
\hline 9 & Clear-cell & - & 2 & $3 b$ & 0 & - \\
\hline 10 & Clear-cell & Azinary, tubulary & 2 & - & - & - \\
\hline 11 & Clear-cell & $\begin{array}{l}\text { Solid, compact, } \\
\text { trabeculary, tubulary }\end{array}$ & 2 & $3 a$ & 0 & $\mathrm{x}$ \\
\hline 12 & Clear-cell & Solid & 3 & - & - & - \\
\hline 13 & Clear-cell & Azinary, tubulary & 2 & - & - & - \\
\hline 14 & Clear-cell & Trabeculary & 2 & - & - & - \\
\hline 15 & Clear-cell & Azinary, tubulary & 2 & - & - & - \\
\hline 16 & Clear-cell & - & 2 & - & - & - \\
\hline 17 & Clear-cell & Aleveolary & 1 & - & - & - \\
\hline
\end{tabular}

${ }^{a}$ Medical Faculty, Institute of Urology, Jena, personal communication.

Friedrich-Schiller-University, Jena. Paraffin sections from each specimen were reviewed by a pathologist and classified histologically according to UICC-TNM classification (1997). All Fuhrmann grading spectra (1-3) are represented (Table I). Additionally, 10 patients ( 3 pRCC and 7 ccRCC) have been examined by CGH-analysis (Table II).

Total RNA extraction. Total RNA was isolated by standard methods using a commercially available RNA isolation kit (Qiagen, Hilden, Germany). A qualitative integrity test of purified total RNA was performed with capillary electrophoresis using a Bioanalyser 2100 (Agilent Technologies, Palo Alto, CA, USA). The amount of RNA was measured with a UV-spectrophotometer.

Microarray experiments. Linear amplification of RNA was performed using a protocol of a previously described method (11). Amplified RNA (aRNA) samples were quantified by spectrophotometry and the quality was reassessed by capillary electrophoresis (Bioanalyser 2001, Agilent). aRNAs were hybridized on the topic-defined PIQOR ${ }^{\mathrm{TM}}$ Immunology microarray (Miltenyi Biotec GmbH, Bergisch Gladbach, Germany). Microarray experiments and data normalization were carried out as previously described (10). On each array the tumor and the corresponding normal tissue of one patient were hybridized. Microarray experiments were performed according to the MIAME guidelines (12).

Real-time RT-PCR. Four primer pairs for CALB1, CD98, CD68 and CD37, as well as one reference gene primer pair for IL1RAP, were used (QuantiTect primer assay, Qiagen). RNA $(2 \mu \mathrm{g})$ was reverse transcribed using SuperScript ${ }^{\mathrm{TM}}$ II reverse transcriptase (Invitrogen) with $100 \mu \mathrm{M}$ random hexamer primers according to the manufacturer's protocol. Real-time RT-PCR was performed with a LightCycler (Roche Molecular Biochemicals, Mannheim, Germany) in capillaries using a commercially available master mix containing HotStartTaq DNA polymerase and SYBR-Green I deoxyribonucleoside triphosphates (QuantiTect SYBR-Green PCR-kit, Qiagen). The following real-time PCR protocol was used for all genes: initial $95^{\circ} \mathrm{C}$ denaturation phase of $15 \mathrm{~min}$ to activate the HotStart enzyme followed by 45 rounds of amplification and quantification $\left(15 \mathrm{sec}\right.$ at $95^{\circ} \mathrm{C} ; 10 \mathrm{sec}$ at $55^{\circ} \mathrm{C} ; 30 \mathrm{sec}$ at $72^{\circ} \mathrm{C}$ ) each with a single fluorescence measurement. The specificity of the desired RT-PCR products was documented using gel electrophoresis and melting curve 
Table II. Overview of the results obtained by CGH-analysis.

\begin{tabular}{ll}
\hline Patient & CGH-analysis \\
\hline 1 & $\operatorname{dim}(\mathrm{y})$, enh(7,17) \\
2 & $\operatorname{dim}(8 \mathrm{p} 11 \mathrm{pter}, \mathrm{Xp}, \mathrm{Y})$, enh(2q22q34, 3p12qter, 9q22pter, 12q24pter) \\
3 & $\operatorname{dim}(1 \mathrm{p}, 20, \mathrm{y})$, enh(17) \\
4 & enh(7,12,17) \\
5 & $\operatorname{enh}(3 \mathrm{q}, 7)$ \\
6 & $\operatorname{dim}(9 \mathrm{q} 22 \mathrm{qter}, 17 \mathrm{p})$, enh(8p12qter, 11q14q23,17q) \\
7 & $\operatorname{dim}(3)$ \\
8 & $\operatorname{dim}(3 \mathrm{p} 21 \mathrm{pter}, 8 \mathrm{p}, 14 \mathrm{q} 21 \mathrm{q} 24)$, enh(5q21qter) \\
9 & $\operatorname{dim}(3 \mathrm{p} 13 \mathrm{pter})$, enh(5q,7) \\
10 & $\operatorname{dim}(3 \mathrm{p}, 4 \mathrm{q} 13 \mathrm{q} 28,7 \mathrm{q}, 10 \mathrm{q} 22,14 \mathrm{q} 13 \mathrm{qter}, 16 \mathrm{q} 17 \mathrm{p})$, enh(2q32pter,5q31qter,6q,13q22qter) \\
11 & $\operatorname{dim}(1 \mathrm{p} 34 \mathrm{qter}, 2 \mathrm{p} 22 \mathrm{pter}, 3,4,6,8,9,13,14,15,18 \mathrm{q}, \mathrm{x}), \mathrm{enh}(16 \mathrm{q} 21 \mathrm{pter}, 19,20)$ \\
12 & $\operatorname{dim}(3 \mathrm{p})$, enh $(4,5,13,21)$ \\
13 & $\operatorname{dim}(3 \mathrm{p} 13 \mathrm{pter}, 14,17,20)$, enh(2,21) \\
14 & $\operatorname{dim}(3 \mathrm{p} 12 \mathrm{pter}, 14,15,17,20, \mathrm{y})$, enh(2,7,11,12,21) \\
15 & $\operatorname{dim}(3 \mathrm{p} 13 \mathrm{pter}, 10 \mathrm{q} 23 \mathrm{qter})$ \\
16 & $\operatorname{dim}(3 \mathrm{p} 14 \mathrm{p} 24,8 \mathrm{p}, 9 \mathrm{q} 21 \mathrm{qter}, 14,17,18, \mathrm{y})$, enh( 8q,9q21pter) \\
17 & $\operatorname{dim}(3 \mathrm{p}, 8 \mathrm{p})$ \\
\hline
\end{tabular}

Table III. Qualitative correlation of microarray and real-time RT-PCR data of four selected genes that discriminate between normal and tumor tissue. ${ }^{a}$

\begin{tabular}{lccc}
\hline Regulated gene & Gene ID & $\begin{array}{c}\text { Number of patients showing } \geq 2 \text {-fold } \\
\text { expression on microarray }\end{array}$ & $\begin{array}{c}\text { Number of patients showing } \geq 2 \text {-fold } \\
\text { expression on LightCycler }\end{array}$ \\
\hline CALB1 & 793 & $7 / 8$ & $10 / 12$ \\
CD98 & 6520 & $8 / 8$ & $10 / 12$ \\
CD37 & 951 & $7 / 8$ & $10 / 12$ \\
CD68 & 968 & $7 / 8$ & $9 / 12$ \\
\hline
\end{tabular}

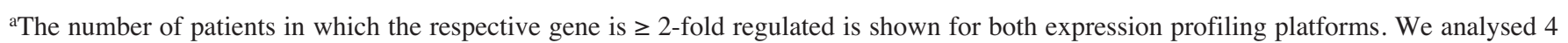
additional patients that have not been examined before on cDNA-microarrays

analysis (LightCyler Software, version 3.5, 2001, Roche Molecular Biochemicals). The 'delta-delta CP method' for comparing relative expression results in real-time PCR was applied (13).

Data preprocessing and clustering analysis. First, both datasets for pRCC and ccRCC were analyzed separately. Genes with missing data in the majority of experiments $(>60 \%)$ were discarded. For further analysis only genes that showed a differential regulation in at least $50 \%$ of the samples (e.g. upregulation in three of six pRCC experiments) and a 1.4-fold regulation in at least one sample were used. From these preliminary datasets, all genes with inconsistent expression profiles, i.e. having a contradictory regulation in two or more out of six (pRCC) and three or more out of 11 (ccRCC) samples, were removed. Genes fulfilling these criteria were referred to as 'regulated equally'. If a sufficient number of genes was identified in these searches, a functional analysis was performed. Additionally, the results of ccRCC and pRCC were then compared to find diagnostic markers common or discriminative for these two variants of RCC.

As the papillary and clear cell datasets were generated using different topic-defined microarrays, the analysis was restricted to the subset of 562 PIQOR probes present on both microarrays. For statistical analysis of the microarrays, a twoclass unpaired statistical analysis of microarrays (SAM) was applied to the dataset assuming a false-discovery rate of zero (14). Because SAM is not effective with missing data points we performed a two-step approach: First, genes with missing data in the majority of experiments $(>60 \%)$ were discarded. Subsequently, any missing values in the remaining genes were estimated by 'N-nearest neighbour imputation' (15). The data preprocessing resulted in two datasets: one containing genes that were equally expressed by both tumor types and one containing genes differentally expressed. Unsupervised hierarchical cluster analysis (Average Linkage Clustering) 
Table IV. Genes identified to be regulated, but not previously described.

\begin{tabular}{lc}
\hline Gene name & Gene ID \\
\hline TGFBI & 7040 \\
TRADD & 8717 \\
FOSL2 & 2355 \\
HSP70.2 & 3303 \\
LTN & 6375 \\
MDC9 & 8754 \\
CSF1R & 1436 \\
BAFF & 10673 \\
CD68 & 968 \\
CD37 & 951 \\
\hline
\end{tabular}

was carried out using 'Cluster' and 'Tree View' programs according to Eisen (16).

Functional annotation analysis. Both datasets for equally and differentially regulated genes in both tumor subtypes were subjected to a pathway annotation analysis by using Miltenyi's TreeRanker system (Miltenyi Biotec GmbH). This software system analyses groups of genes for a statistically significant enrichment of biological pathway annotation terms. Term enrichment relative to the expected background distribution was scored using Fisher's exact test. Two basic features set TreeRanker apart from similar approaches published recently (17-19). First, annotations derived from fundamentally different sources (Gene Ontology, gene families, sequence motifs, chromosomal proximity, literature keywords, etc.) can be scored simultaneously for each given group of genes, resulting in a joint statistical significance value. Second, the scoring approach can be applied to entire hierarchical gene trees, scoring individual sub-branches and reporting only non-redundant instances of significant annotation enrichment in specific clusters. In this mode, a Bonferroni-correction is applied to the significance values to account for the multipletesting situations. Genes up or downregulated in both tumor subtypes were subjected to further analysis with Interaction Explorer Software Pathway Assist (version 2.5, Stratagene). The ResNet database (as of August 30, 2005) was searched for common cellular processes and protein complexes.

\section{Results}

Gene expression analysis of $p R C C$. From the 1070 available genes present on the microarray, $602(55.7 \%)$ genes were found to be more than 2-fold differentially expressed in at least one patient. We found a total of 10 genes to be overexpressed that have not been described for pRCC (Table IV). Additionally, we found a slight, but nevertheless consistent, overexpression for the genes TNFR1 and TNFRSF10B. The already known upregulation of the genes TIMP1 and THBS2 was confirmed $(20,21)$.

The gene with the most prominent downregulation in the pRCCs is PLCG2. We also found CD98, CCL13 and CXCL14,

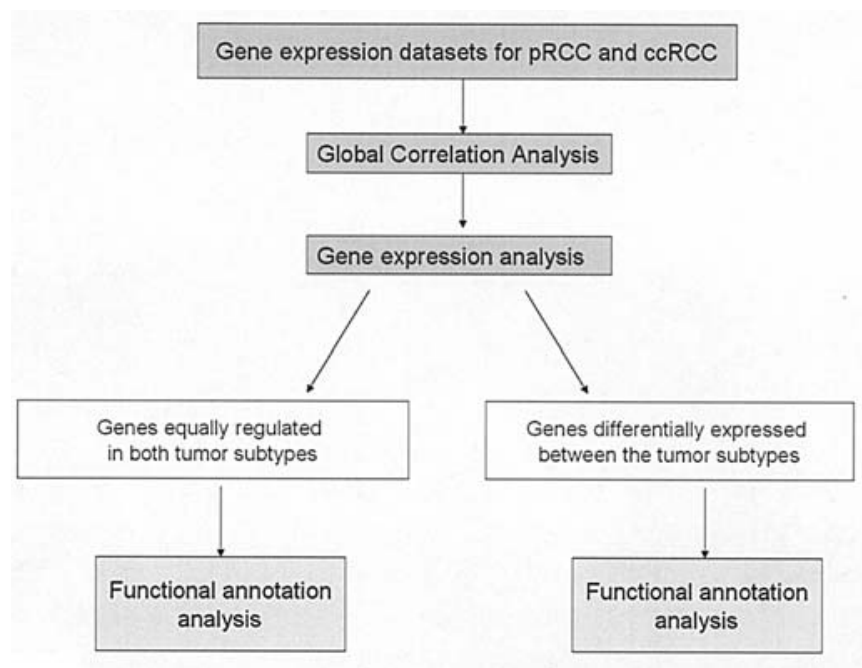

Figure 1. Overview of the analysis design carried out in this study.

and CALB1 to be downregulated in most samples. Genes with a more moderate but nevertheless quite continuous downregulation include HSPA1A, DCN, MATRIXGLA, MGST2, TTRAP, CD164, PLAT, SOD1, and CXCL12.

Confirmation of selected genes differentially expressed in pRCC via real-time $R T-P C R$. A randomly selected subset of genes (CD37, CALB1, CD96, CD68) found to be differentially expressed in pRCC was confirmed via real-time RT-PCR (Table III). In order to compare the quantified mRNA molecules of the candidate genes in the relative expression ratio model, IL1RAP was chosen as the reference gene because it showed no significant regulation on the microarrays. The real-time RT-PCR efficiency was set to $2(\mathrm{E}=2.0)$ for all factors. The standard deviation in all experiments performed is between 0 and $3.38 \%$. The regulations found by cDNAmicroarrays were confirmed for all four genes. In order to independently validate these regulations, we also performed real-time RT-PCR on four additional patients that had not been previously examined on microarrays. The detected regulations were again confirmed.

Comparative functional analysis. For the comparative functional analysis we combined the dataset for gene expression in pRCC with a dataset for gene expression in ccRCC. First, we performed a global correlation analysis to find out wether the gene expression profiles of patients belonging to the same tumor subtype cluster together. Second, we searched each dataset for genes similarly regulated in patient samples and third, we performed a functional analysis if a sufficient number of genes regulated was identified (Fig. 1).

Global correlation analysis. We assessed the correlation between the expression profiles of the clear cell and papillary dataset (Fig. 2). For this purpose, we used all genes that were found to be expressed in at least $55 \%$ of the patient samples. The expression profiles of both datasets satisfactorily cluster in clearly separable groups. Two patients of the ccRCC dataset show a very divergent expression profile, neither matching the ccRCC samples nor the pRCC. Additionally, one 
Table V. Overview of genes up- or downregulated in both examined RCC types. ${ }^{\text {a }}$

\begin{tabular}{|c|c|c|c|c|c|}
\hline \multicolumn{3}{|c|}{ Genes upregulated in both tumor types } & \multicolumn{3}{|c|}{ Genes downregulated in both tumor types } \\
\hline Gene Name & Gene ID & Annotation & Gene Name & Gene ID & Annotation \\
\hline TNFR1 & 7132 & Surface marker & $\mathrm{CCNH}$ & 902 & DNA-metabolism \\
\hline BAFF & 10673 & Surface marker & BAMACAN & 9126 & DNA-metabolism \\
\hline TNFRSF10B & 8795 & Surface marker & HSPA1A/HSPA1B & 3303 & DNA-metabolism/HSP70 \\
\hline PMP22 & 5376 & Surface marker & ANPEP & 290 & Enzyme \\
\hline PDGFRB & 5159 & Surface marker & SOD1 & 6647 & Enzyme \\
\hline CD53 & 963 & Surface marker & FYN & 2534 & Enzyme \\
\hline FCGR1A & 2209 & Surface marker & PLAT & 2811 & Enzyme \\
\hline CD68 & 968 & Surface marker & RAR & 10966 & Enzyme \\
\hline CD70 & 970 & Surface marker & PLCL & 5334 & Enzyme/lipid metabolism \\
\hline CD19 & 930 & Surface marker & PRKCL2 & 5586 & Enzyme/lipid metabolism \\
\hline FCGR2 & $2212 / 2213$ & Surface marker & PRSS11 & 5654 & Enzyme/lipid metabolism \\
\hline CD45 & 5788 & Surface marker & PIK3CB & 5291 & Enzyme/lipid metabolism \\
\hline IL8 & 3576 & m.a. & PLCG2 & 5336 & Enzyme/lipid metabolism \\
\hline IL16 & 3603 & m.a. & HSPA8 & 3312 & HSP70 \\
\hline TUBA & 10376 & m.a. & HSPA9 & 3313 & HSP70 \\
\hline CASP4 & 837 & m.a. & CTGF & 1490 & Lipid metabolism \\
\hline BID & 637 & m.a. & CYR61 & 3491 & Lipid metabolism \\
\hline PLGF & 5228 & m.a. & COL18A1 & 80781 & m.a. \\
\hline CSK & 1445 & m.a. & COL6A1 & 1291 & m.a. \\
\hline CX3CR1 & 1524 & m.a. & LTBP4 & 8425 & m.a. \\
\hline SPARC & 8404 & m.a. & $\mathrm{DCN}$ & 1634 & m.a. \\
\hline \multirow[t]{2}{*}{ STAT1 } & 6772 & m.a. & ATF5 & 22809 & m.a. \\
\hline & & & BACH1 & 571 & m.a. \\
\hline
\end{tabular}

${ }^{\mathrm{a}} \mathrm{m} . \mathrm{a} .$, multiple annotation.

experiment belonging to the pRCC dataset correlates better to the ccRCC dataset. A closer inspection of patient data (histology, age, gender and other pathologically relevant data) did not reveal possible reasons for this outlier.

Regulated genes in $p R C C$ and $c c R C C$. The comparative functional analysis revealed genes to be regulated in both tumor subtypes in the same direction (equally) as well as differentially. A total of 45 genes was identified to be regulated equally in both $\mathrm{RCC}$ types (Table V). Among the upregulated genes in both tumor types was a significantly relevant group of 12 genes which are surface markers. The downregulated genes in both tumor types were mostly enzymes (10 genes) or genes involved in lipid and carbohydrate metabolism (Table V).

The differentially expressed genes in both tumor subtypes comprise thirteen genes (TNFR2, RPS7, CD151, VCAM1, LUMAN, ATF4, HEVIN, CCND1, VEGF, CD40, PLGF, CD70 and FOSL2) that are upregulated in the ccRCC patients and downregulated in the pRCC patients (Fig. 3). This gene set distinguishes between the tumor subtypes applying a falsediscovery rate of zero to the combined dataset. Only one gene (FOSL2) was found to be upregulated in pRCC and downregulated in ccRCC. CD70 as well as PLGF are strongly upregulated (32-fold and 20.8-fold, respectively) in all ccRCC samples, but only weakly (1.2-fold and 1.1-fold, respectively) in the pRCC samples. FOSL2/FRA2 and HEVIN exhibit the most contrary expression between the tumor subtypes.

Functional annotation analysis. The 45 equally regulated genes (22 upregulated and 23 downregulated in both tumor subtypes) were subjected to a functional annotation analysis (Table V). The aim was to search for common cellular processes enabled in RCC. The relations found for the upregulated genes are shown in Fig. 4 and for the downregulated genes in Fig. 5. The downregulated genes in both tumor types are mainly enzymes and genes related to lipid and carbohydrate metabolism (Table V). Several downregulated genes (CTGF, ATF5, SOD1, HSPA8, FYN, COL18A1, HSPA1a) were found that are related to apoptosis or cell death (Fig. 6). Among the upregulated genes in both tumor subtypes, we found a statistically significant group of 12 upregulated surface markers. Five out of the 12 genes (TNFRSF10B, CD70, TNFR1, PDGFRB and BAFF) are directly related to immune response, apoptosis and cell death (Fig. 6).

\section{Discussion}

Renal cell carcinoma (RCC) is known to prevent an immune response via induction of apoptosis in T-cells. Even though various gene expression studies have been carried out, only a few genes, such as FasL, are known to play a role in these 

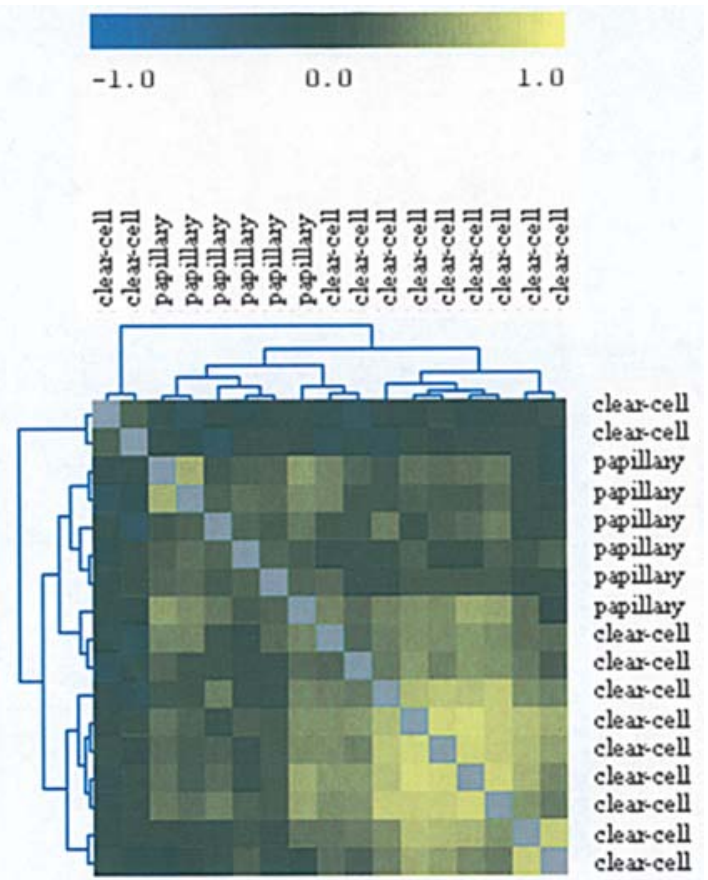

Figure 2. Global inter-experiment correlation analysis of genes present on both topic-defined PIQOR microarray compositions. Yellow color indicates good correlation between two expression profiles, while black squares represent poor or even anti-correlation (blue).

mechanisms (4). The discovery of more genes that are involved in this mechanism would lead to the development of more effective immunotherapeutic approaches.

Gene expression studies carried out for RCC using microarray technologies have already been successfully applied toward the identification of cancer-associated expression patterns $(8,9)$. Several studies have also been published using transcriptional profiling to identify genes that are differentially regulated $(6,22)$. However, most of these studies are often restricted solely to changes in gene expression and do not address functional patterns. So far no functional profiling analysis has been carried out for RCC.

The aim of the present study was to compare the gene expression of the RCC subtypes pRCC and ccRCC. First, we analysed the gene expression of both tumor subtypes. Using Miltenyi's TreeRanker system (Miltenyi Biotec GmbH) we identified clusters of interacting genes that were coordinately or differentially up or downregulated. We then identified a dataset with differentially expressed genes (a total of 13 genes) between pRCC and ccRCC (Fig. 3). Furthermore, we found a dataset with 45 genes regulated equally in both tumor subtypes (Table V). Functional profiling revealed differences and similarities in the gene expression patterns of both tumor subtypes.

Confirmation of microarray data. To confirm our data, we compared the differentially expressed genes of this study with earlier pRCC studies. Our results were consistent with those studies that had overlapping datasets. For example, our results match previously published data for the genes THBS2, C-Kit, GRO-1, TIMP1, TIMP2, CXCR4, and IGFBP6 $(20,21,23,24)$. Additionally, we validated the cDNA microarray results via real-time RT-PCR for randomly selected genes. We found a strong correlation between the microarray and real-time RTPCR analyses for all examined genes. In order to confirm the expression patterns found independently from the microarrays, we subjected four separate (pRCC) patient samples that had not been analysed on microarrays to a real-time RT-PCR analysis. We again confirmed the expression patterns found with the cDNA microarrays.

Genes differentially expressed between the tumor subtypes. The first dataset analysed contained 13 genes that are differentially expressed between both tumor subtypes. This dataset served to discriminate between the two RCC subtypes. FRA2/FOSL2 and HEVIN exhibited the most contrary expression between the examined tumor subtypes. FRA2 was strongly downregulated in ccRCC whereas HEVIN was downregulated in

\begin{tabular}{|lll}
\hline & & \\
\hline-3.0 & 0.0 & 3.0
\end{tabular}

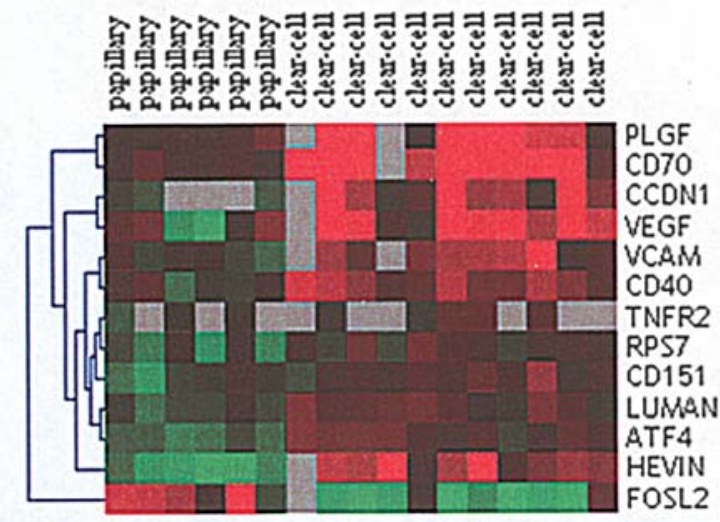

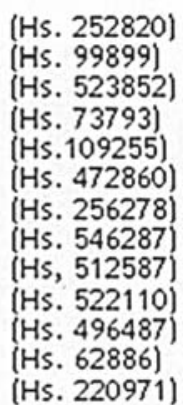

Figure 3. Overview of genes discriminating between the papillary and clear cell RCC datasets. (Unsupervised one-dimensional clustering of log ${ }^{2}$ data using the 'Euclidean distance' metric). 


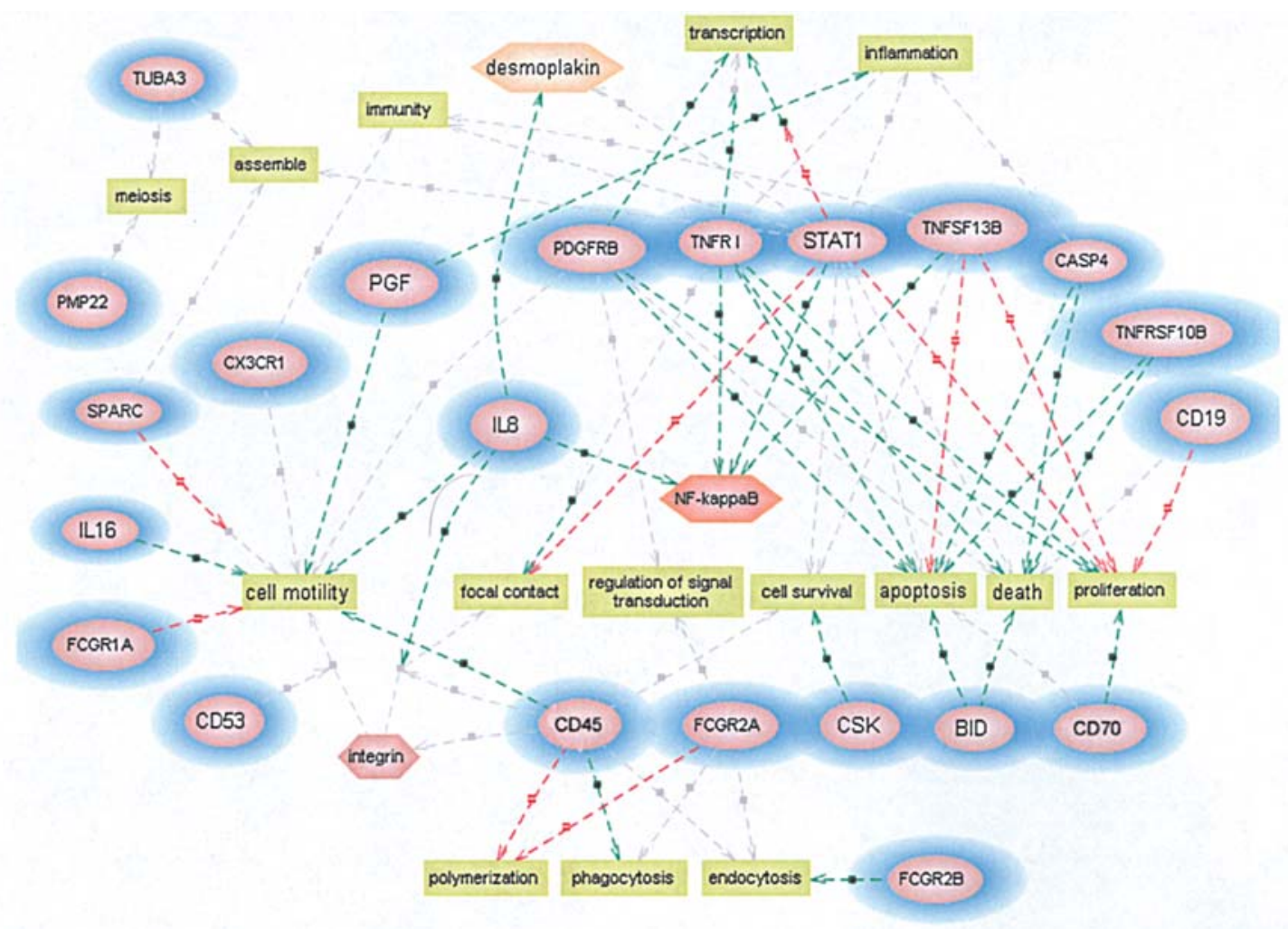

Figure 4. Network analysis of the upregulated genes in both tumor subtypes (blue halo) to identify common cellular processes (yellow boxes) and protein complexes (hexagons). Most of the processes involved are related to apoptosis or cell death.

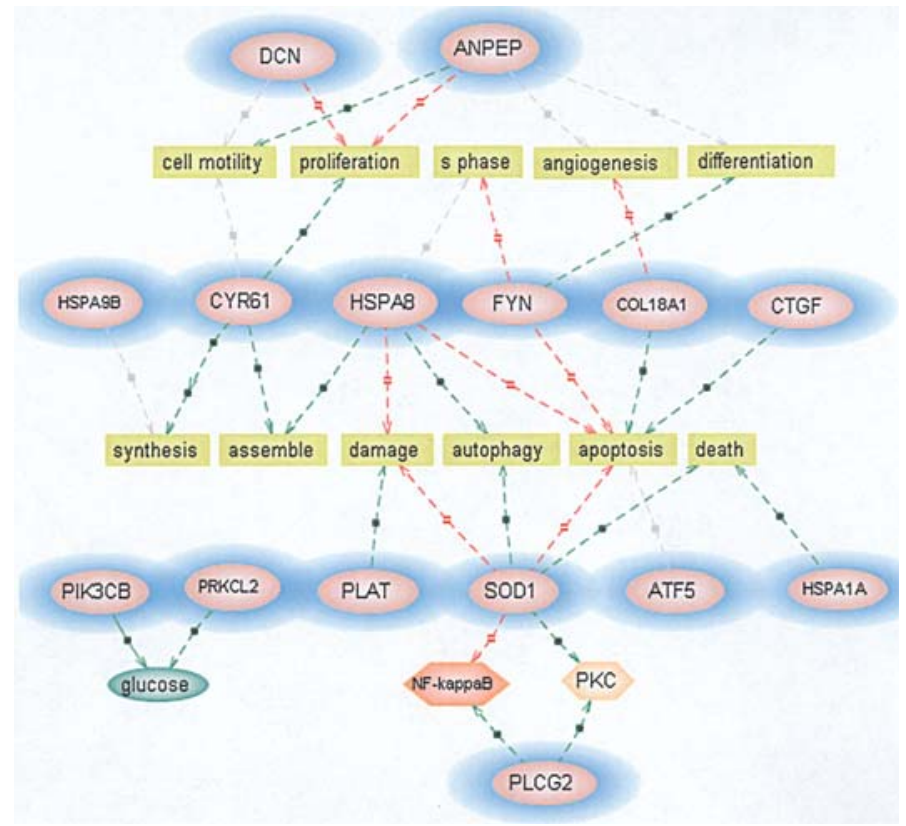

Figure 5. Network analysis of the downregulated genes in both tumor subtypes (blue halo) to identify common cellular processes (yellow boxes) and protein complexes (hexagons).

pRCC. In some cases, expression of the FOS gene has also been associated with apoptotic cell death (25). HEVIN is an extra-cellular matrix-associated secreted glycoprotein belonging to the secreted protein acidic and rich in cysteine (SPARC) family of matricellular proteins. We identified a

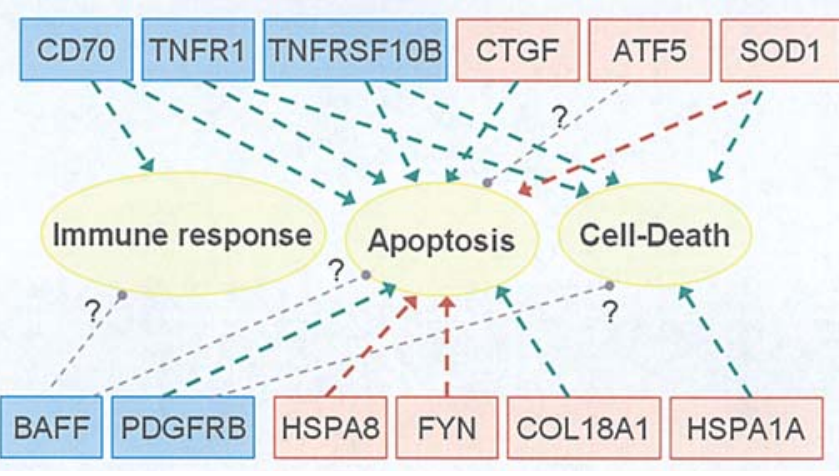

Figure 6. Schematic representation of genes commonly expressed by both tumor subtypes involved in RCC immunogenicity (blue, upregulated genes; red, downregulated genes; yellow, affected processes; green arrow, activation; red arrow, inhibition; grey arrow, unkown influence).

number of genes upregulated in ccRCC but not in pRCC that play a role in cell-cell recognition, such as VEGFA and VCAM1. Interestingly, CD40, a gene upregulated in ccRCC, was also involved in an immune response by regulating B-cell proliferation. However, we also found an upregulation for the gene TNFR2 that mediates most of the metabolic effects of TNF $\alpha$ including blocking its apoptotic functions.

The findings that the genes RPS7, CD151, LUMAN, and ATF4 were upregulated in ccRCC but not in pRCC is congruent with previous studies that report discrete microarray patterns in RCC subtypes $(6,22,24)$. Our study found additional differences in regulation between the two tumor subtypes for CD70, PLGF, and CCND1. The gene CCND1 
showed a rather heterogeneous expression pattern and was not consistently expressed in all patient samples analysed. Meanwhile, CD70 and PLGF were strongly upregulated in ccRCC but nearly unaffected in the pRCC dataset. A closer look at the induction revealed that while both genes were only slightly induced in pRCC, they were more than 20-fold upregulated in ccRCC. These ambiguous results for CD70 and PLGF might not only reflect the heterogeneity of the cell population in the examined tumor tissues, but also patientspecific differences in gene expression. Consequently we included these genes in both the equally and differentially expressed gene datasets.

Genes equally expressed in the tumor subtypes. The 45 genes equally expressed (22 upregulated and 23 downregulated) by both tumor types were analysed via functional profiling (Table V).

Downregulation in both tumor subtypes. The majority of genes downregulated in both tumor types were enzymes (10 genes) and genes involved in lipid and carbohydrate metabolism (7 genes) (Table V). The functional analysis revealed their involvement in several cellular processes such as cell motility, proliferation and angiogenesis. Most importantly, several of these commonly downregulated genes were found to be involved in mechanisms concerning apoptosis and cell death (FYN, COL18A1, CTGF, SOD1, ATF and HSPA1A) (Fig. 5).

Upregulation in both tumor subtypes. Most of the upregulated genes in both tumor types were surface markers (Table V and Fig. 4). Since these markers play a key role in cell-cell communication and are crucial for adaptive and antigen-directed immunity we constrained our analysis to identifying the underlying functional relations between them (Figs. 4 and 6). Among the genes found to be regulated are FCGR1A, FCGR2, and CD45. They are known to be regulators of $\mathrm{T}$-cell antigen receptor signaling and are therefore directly involved in an anti-immune response of RCC (26). CD19 may be involved in the growth regulation of B-cells and CD68 could play a role in phagocytic activities of tissue macrophages, both in intra-cellular lysosomal metabolism and extracellular cell-cell and cell-pathogen interactions. The gene PMP22 might be involved in growth regulation whereas PDGFRB is a receptor that binds specifically to PDGFB and has a tyrosine-protein kinase activity.

Most interestingly, four of the twelve upregulated surface markers, TNFRSF10B, CD70, TNFR1, and BAFF, belong to the tumor necrosis factor/tumor necrosis factor receptor related superfamily, a group of proteins that function as communicators between cells. Additionally, a fifth surface marker, PDGFRB, has been found to be upregulated in both tumor subtypes. All five of these proteins mentioned before can mediate apoptosis or are linked directly to an immune response via their ability to regulate the reaction of lymphocytes to pathogens (Fig. 6) (27).

It has already been speculated that CD70 could play an important role in RCC immune escape, more so since Chahlavi et al showed that the overexpression of CD70 in glioblastoma leads to T-cell death (28). Given the strong upregulation of CD70 in ccRCC and the accumulation of upregulated immunogenic genes in RCC, we think that genes belonging to the TNF receptor family could play a key role in RCC immune escape. However, the role of CD70 as a possible mediator of T-cell death for RCC should be investigated in more detail.

Our approach provides information on the functional similarities of the two most common RCC types. The functional comparison of both tumor subtypes point on the one hand to a remarkable difference between pRCC and ccRCC. On the other hand, both tumor types seem to utilize similar mechanisms and patterns to successfully escape the human immune system. We found a subset of genes to be equally regulated by both tumor subtypes, which are all related to apoptosis or T-cell regulation. These findings elucidate genes possibly involved in RCC immune escape strategies that should be investigated in more detail. Some of these genes could potentially be new targets for novel therapeutic agents.

\section{Acknowledgements}

This work was supported by a Grant of the German Federal Ministry of Education and Research (BMBF) and the Interdisciplinary Center for Clinical Research (ICCR), Jena. Furthermore we thank Professor Hans-Peter Saluz and Grit Mrotzek from the Hans Knöll Institute, Jena for their cooperation. We also thank Melanie Diegmann for her assistance in the gene expression analysis and Dr Ryan K. Oyama for critically proof-reading the manuscript.

\section{References}

1. Eble JN: WHO-classification of tumors. In: Pathology and genetics of tumors of the urinary system and male genital organs. Sauter G, Epstein J and Sesterhenn I (eds). IARC Press, Lyon, 2004

2. Storkel S, Eble JN, Adlakha K, Amin M, Blute ML, Bostwick DG, Darson M, Delahunt B and Iczkowski K: Classification of renal cell carcinoma: Workgroup No. 1. Union Internationale Contre le Cancer (UICC) and the American Joint Committee on Cancer (AJCC). Cancer 80: 987-989, 1997.

3. Kovacs G, Akhtar M, Beckwith BJ, Bugert P, Cooper CS, Delahunt B, Eble JN, Fleming S, Ljungberg B, Medeiros LJ, Moch H, Reuter VE, et al: The Heidelberg classification of renal cell tumours. J Pathol 183: 131-133, 1997.

4. Uzzo RG, Rayman P, Kolenko V, Clark PE, Bloom T, Ward AM, Molto L, Tannenbaum C, Worford LJ, Bukowski R, Tubbs R, Hsi ED, et al: Mechanisms of apoptosis in T cells from patients with renal cell carcinoma. Clin Cancer Res 5: 1219-1229, 1999.

5. Hedenfalk I, Duggan D, Chen Y, Radmacher M, Bittner M, Simon R, Meltzer P, Gusterson B, Esteller M, Kallioniemi OP, Wilfond B, Borg A, et al: Gene-expression profiles in hereditary breast cancer. N Engl J Med 344: 539-548, 2001.

6. Boer JM, Huber WK, Sultmann H, Wilmer F, von HA, Haas S, Korn B, Gunawan B, Vente A, Fuzesi L, Vingron M and Poustka A: Identification and classification of differentially expressed genes in renal cell carcinoma by expression profiling on a global human 31,500-element cDNA array. Genome Res 11: 1861-1870, 2001.

7. Draghici S, Khatri P, Martins RP, Ostermeier GC and Krawetz SA: Global functional profiling of gene expression. Genomics 81: 98-104, 2003.

8. Arlt D, Huber W, Liebel U, Schmidt C, Majety M, Sauermann M, Rosenfelder H, Bechtel S, Mehrle A, Bannasch D, Schupp I, Seiler M, et al: Functional profiling: from microarrays via cellbased assays to novel tumor relevant modulators of the cell cycle. Cancer Res 65: 7733-7742, 2005.

9. Donninger $\mathrm{H}$, Bonome T, Radonovich M, Pise-Masison CA, Brady J, Shih JH, Barrett JC and Birrer MJ: Whole genome expression profiling of advance stage papillary serous ovarian cancer reveals activated pathways. Oncogene 23: 8065-8077, 2004. 
10. Diegmann J, Junker K, Gerstmayer B, Bosio A, Hindermann W, Rosenhahn J and von EF: Identification of CD70 as a diagnostic biomarker for clear cell renal cell carcinoma by gene expression profiling, real-time RT-PCR and immunohistochemistry. Eur J Cancer 41: 1794-1801, 2005.

11. Eberwine J: Amplification of mRNA populations using aRNA generated from immobilized oligo(dT)-T7 primed cDNA. Biotechniques 20: 584-591, 1996.

12. Brazma A, Hingamp P, Quackenbush J, Sherlock G, Spellman P, Stoeckert C, Aach J, Ansorge W, Ball CA, Causton HC, Gaasterland T, Glenisson P, et al: Minimum information about a microarray experiment (MIAME)-toward standards for microarray data. Nat Genet 29: 365-371, 2001.

13. Livak KJ and Schmittgen TD: Analysis of relative gene expression data using real-time quantitative PCR and the 2(Delta Delta C(T)) Method. Methods 25: 402-408, 2001.

14. Tusher VG, Tibshirani R and Chu G. Significance analysis of microarrays applied to the ionizing radiation response. Proc Natl Acad Sci USA 98: 5116-5121, 2001.

15. Troyanskaya O, Cantor M, Sherlock G, Brown P, Hastie T, Tibshirani R, Botstein D and Altman RB: Missing value estimation methods for DNA microarrays. Bioinformatics 17: 520-525, 2001.

16. Eisen MB, Spellman PT, Brown PO and Botstein D: Cluster analysis and display of genome-wide expression patterns. Proc Natl Acad Sci USA 95: 14863-14868, 1998.

17. Vencio RZ, Koide T, Gomes SL and Pereira CA: Bay GO: Bayesian analysis of ontology term enrichment in microarray data. BMC Bioinformatics 7: 86, 2006.

18. Boyle EI, Weng S, Gollub J, Jin H, Botstein D, Cherry JM and Sherlock G: GO:TermFinder - open source software for accessing Gene Ontology information and finding significantly enriched Gene Ontology terms associated with a list of genes. Bioinformatics 20: 3710-3715, 2004.

19. Xu Q and Shaulsky G: GOAT: An R Tool for Analysing Gene Ontology trade mark Term Enrichment. Appl Bioinformatics 4: 281-283, 2005.

20. Kallakury BV, Karikehalli S, Haholu A, Sheehan CE, Azumi N and Ross JS. Increased expression of matrix metalloproteinases 2 and 9 and tissue inhibitors of metalloproteinases 1 and 2 correlate with poor prognostic variables in renal cell carcinoma. Clin Cancer Res 7: 3113-3119, 2001.
21. Gerritsen ME, Peale FV Jr and Wu T: Gene expression profiling in silico: relative expression of candidate angiogenesis associated genes in renal cell carcinomas. Exp Nephrol 10: 114-119, 2002.

22. Higgins JP, Shinghal R, Gill H, Reese JH, Terris M, Cohen RJ, Fero M, Pollack JR, van de RM and Brooks JD: Gene expression patterns in renal cell carcinoma assessed by complementary DNA microarray. Am J Pathol 162: 925-932, 2003.

23. Petit A, Castillo M, Santos M, Mellado B, Alcover JB, Mallofre C: KIT expression in chromophobe renal cell carcinoma: comparative immunohistochemical analysis of KIT expression in different renal cell neoplasms. Am J Surg Pathol 28: 676-678, 2004.

24. Takahashi M, Yang XJ, Sugimura J, Backdahl J, Tretiakova M, Qian CN, Gray SG, Knapp R, Anema J, Kahnoski R, Nicol D, Vogelzang NJ, et al: Molecular subclassification of kidney tumors and the discovery of new diagnostic markers. Oncogene 22: 6810-6818, 2003.

25. Shirsat NV and Shaikh SA: Overexpression of the immediate early gene fra- 1 inhibits proliferation, induces apoptosis, and reduces tumourigenicity of c6 glioma cells. Exp Cell Res 291: 91-100, 2003

26. Nakamura A, Akiyama K and Takai T: Fc receptor targeting in the treatment of allergy, autoimmune diseases and cancer. Expert Opin Ther Targets 9: 169-190, 2005.

27. Locksley RM, Killeen N and Lenardo MJ: The TNF and TNF receptor superfamilies: integrating mammalian biology. Cell 104: 487-501, 2001.

28. Chahlavi A, Rayman P, Richmond AL, Biswas K, Zhang R, Vogelbaum M, Tannenbaum C, Barnett $\mathrm{G}$ and Finke JH: Glioblastomas induce T-lymphocyte death by two distinct pathways involving gangliosides and CD70. Cancer Res 65: 5428-5438, 2005. 